\title{
Comportamiento productivo de pollos que se alimentaron con granos tostados de Cajanus cajan
}

\author{
Herrera, G.S.M. ${ }^{1}$; Díaz, C.A. ${ }^{2}$; Macías, V. J. ${ }^{\text {; }}$ Solís, B.T. ${ }^{1}$ y Muñoz, R.G. ${ }^{1}$
}

'Universidad Técnica de Quevedo (UTEQ). Facultad de Ciencias Pecuarias. Quevedo. Los Ríos. Ecuador.

${ }^{2}$ Universidad de Guayaquil (UG). Facultad de Medicina Veterinaria y Zootecnia. Guayaquil. Guayas. Ecuador.

Palabras claVe adicionales

Consumo.

Conversión.

Avicultura

Ganancia de peso.

\section{ADDITIONAL KEYWORDS}

Consumption.

Conversion.

Poultry

Weight gain.

\section{RESUMEN}

Se comparó el comportamiento productivo de 144 pollos de ceba $(72$ por sexo, con 1 día de edad y $50 \mathrm{~g}$ de peso) que se alimentaron con balanceado comercial (grupo control) o la sustitución de una parte del maíz y la soya, por harina de semillas tostadas de gandul (C. cajan) (6\%). Los tres tratamientos experimentales ( 36 animales por grupo) fueron: $\mathrm{I}, 120^{\circ} \mathrm{C}$ por $12 \mathrm{~min}$; II, $130^{\circ} \mathrm{C}$ por $15 \mathrm{~min}$; y III: $140^{\circ} \mathrm{C}$ por $18 \mathrm{~min}$. Los animales se alojaron en instalaciones rústicas y se criaron en piso, con libre acceso al agua y al alimento las 24 horas del día e iluminación nocturna. Se pesaron cada siete días, 7:30 horas, antes de la ingestión del alimento. El mayor $(p<0,05)$ consumo total de materia seca $(7744,90 \mathrm{~g})$, ganancia media diaria de peso vivo acumulada por animal en la crianza $(62,79 \mathrm{~g})$, peso vivo final por animal $(3566,43$ g) y eficiencia en el uso de la proteína y la energía se obtuvo en el tratamiento I. No hubo diferencias en la conversión de alimentos y todas las dietas cubrieron los requerimientos nutricionales. Se demostró que es posible mejorar el comportamiento productivo de pollos de ceba en inicio y crecimiento-ceba, con la sustitución del $6 \%$ de la soya y el maíz de la ración por harina de semillas tostadas de C. cajan a $120^{\circ} \mathrm{C}$ por $12 \mathrm{~min}$.

Productive performance of chickens fed with Cajanus cajan (gandul) roasted grains SUMMARY

Productive performance of 144 broiler chickens (72 by sex, age 1 day to $50 \mathrm{~g}$ weight) were fed commercial feed (control group) or replacement of part of the corn and soybean meal were compared by roasted seeds of $C$. cajan $(6 \%)$. The three experimental treatments (36 animals per group) were: I, $120^{\circ} \mathrm{C}$ for $12 \mathrm{~min}$; II, $130^{\circ} \mathrm{C}$ for $15 \mathrm{~min}$; and III: $140^{\circ} \mathrm{C}$ for $18 \mathrm{~min}$. The animals were housed in rustic installation and bred in floor, with free access to water and food, 24 hours a day and night illumination. They were weighed every seven days, 7:30 am, prior to ingestion of food. The higher $(p<0.05)$ total dry matter intake $(7744.90 \mathrm{~g})$, average daily live weight gain accumulated by animal breeding $(62.79 \mathrm{~g})$, final live weight per animal (3566.43 g) and efficiency of the protein and energy used was obtained in treatment I. There was no difference in feed conversion and covered all diets nutritional requirements. Demonstrated that to be possible to improve the productive performance of broiler chickens in starting and growing-fattening, with the replacement of $6 \%$ from soy and corn flour ration of roasted seeds of $\mathrm{C}$. cajan at $120^{\circ} \mathrm{C}$ for $12 \mathrm{~min}$.the breeding program.

\section{INFORMACIÓN}

Cronología del artículo.

Recibido/Received: 05.08.2015

Aceptado/Accepted: 12.02 .2016

On-line: 11.06 .2016

Correspondencia a los autores/Contact e-mail:

mallyhe55@hotmail.com

\section{INTRODUCCIÓN}

Cajanus cajan L. se puede utilizar, para la sustitución de una parte de la soya y el maíz de la dieta de los pollos (Polyana et al., 2014 y Sourokou, 2014). Esta planta oriunda de África, es rica en proteína (Mula y Saxena, 2010; Barboza, 2012 y Rufini, 2014). Sin embargo, posee factores antinutricionales (FAN), que reducen la palatabilidad e inhiben la digestión y absorción de nutrientes, la mayoría, termo lábiles (Elizalde et al., 2009), como las lectinas y los inhibidores de proteasas (León et al., 2011; Navarro et al., 2014 y Bracho et al., 2014) y el tratamiento térmico podría mejorar el comportamiento productivo de pollos de engorde. El objetivo del trabajo fue comparar el comportamiento productivo de pollos de ceba que se alimentaron con balanceado comercial o la sustitución de una parte del maíz y la soya, por harina de semillas de C. cajan, tostadas a diferentes temperaturas $\mathrm{y}$ tiempos.

\section{MATERIALES Y MÉTODOS}

Se realizó en Quevedo, Los Ríos, Ecuador, a 01 $06^{\prime}$ latitud sur y $79^{\circ} 29^{\prime}$ latitud oeste, 75 metros sobre el 
nivel del mar, temperatura promedio anual de $24,70^{\circ} \mathrm{C}$, humedad relativa del $87 \%$, precipitación promedio anual de $2613 \mathrm{~mm}$, heliofanía anual de 886 horas y suelo franco arcilloso.

Se utilizaron 144 pollos de ceba, en un diseño de bloques al azar, distribuidos homogéneamente según el peso, en cuatro bloques, 72 animales por sexo y 36 por grupo, con seis réplicas, de seis aves cada una. Se trabajó en las fases inicial, de 0-4 semanas de edad (fase I) y de crecimiento-ceba, de 5-8 semanas (fase II). Se les ofertó la ración una vez al día, con libre acceso al agua y al alimento. Las semillas de gandul (Cajanus cajan) se tostaron en recipientes metálicos calentados al carbón. Se removieron continuamente y se midió la temperatura, hasta alcanzar los grados, durante los minutos (min), de cada tratamiento, en los grupos experimentales $\left(\mathrm{I}, 120^{\circ} \mathrm{C}\right.$ por $12 \mathrm{~min}$; II, $130^{\circ} \mathrm{C}$ por $15 \mathrm{~min}$; y III: $140^{\circ} \mathrm{C}$ por $\left.18 \mathrm{~min}\right)$. Las semillas se molieron con un molino de martillo, para elaborar las harinas $(6 \%$ de inclusión en los grupos experimentales). Se determinaron la materia seca (MS), proteína cruda (PC), energía metabolizable (EM), fibra cruda $(\mathrm{FC})$, calcio $(\mathrm{Ca}) \mathrm{y}$ fósforo (AOAC, 2012) de las dietas iso-energéticas e iso-proteicas, para cada fase (tabla I).

Se vacunaron contra el virus Newcastle y se alojaron en instalaciones rústicas. Se criaron en piso, con una cama de viruta de $15 \mathrm{~cm}$. Se pesaron individualmente, cada siete días. Se midió el consumo de alimento, se calculó la conversión alimentaria y la eficiencia en la utilización de la energía y la proteína, para la ganancia de peso que se obtuvo. No hubo mortalidad durante la investigación. Los datos se analizaron por el software SAS (Statistical Analysis System), versión 9.3 (2013), y se utilizó la prueba de múltiples rangos de Tukey, para la comparación de las medias, en el análisis de varianza (ANOVA).

\section{RESULTADOS}

El mayor $(p<0,05)$ consumo $(7744,90 \mathrm{~g})$, peso vivo (3 566,43 g), ganancia total $(62,79 \mathrm{~g}$ ) y eficiencia en el uso de la energía y la proteína se obtuvieron con semillas tostadas de C. cajan a $120^{\circ} \mathrm{C}$ por $12 \mathrm{~min}$. No hubo

Tabla I. Composición de las dietas por fase de crecimiento de pollos de ceba que se alimentaron con harina de semillas tostadas de C. cajan e indicadores bromatológicos (Composition of diets for growth phase of broilers that were fed with flour roasted seeds of $C$. cajan and bromatological indicators).

\begin{tabular}{|c|c|c|c|c|c|c|c|c|}
\hline \multirow{2}{*}{$\begin{array}{l}\text { Ingredientes (\%) } \\
\text { Fases de la crianza }\end{array}$} & \multicolumn{2}{|c|}{ Control } & \multicolumn{6}{|c|}{ Harinas de C. cajan (gandul) } \\
\hline & I & II & I & II & 1 & II & I & II \\
\hline Maíz, harina & 62,12 & 67,62 & 58,09 & 61,58 & 58,23 & 61,58 & 58,12 & 61,58 \\
\hline Soya, harina & 16,97 & 10 & 15,28 & 10 & 15 & 10 & 15,12 & 10 \\
\hline Gandul, harina & 0 & 0 & 6 & 6 & 6 & 6 & 6 & 6 \\
\hline Palma, aceite & 1,80 & 3 & 1,50 & 3 & 1,50 & 3 & 1,80 & 3 \\
\hline Pescado, harina & 8,50 & 8,50 & 8,50 & 8,50 & 8,50 & 8,50 & 8,50 & 8,50 \\
\hline Alfalfa, harina & 5 & 5 & 5 & 5 & 5 & 5 & 5 & 5 \\
\hline Arroz, polvo & 3 & 3,12 & 3 & 3,12 & 3,12 & 3,12 & 3 & 3,12 \\
\hline Fosfato di-cálcico & 0,62 & 0,70 & 0,70 & 0,70 & 0,70 & 0,70 & 0,38 & 0,70 \\
\hline Pre-mezcla ${ }^{1}$ & 0,25 & 0,25 & 0,25 & 0,25 & 0,25 & 0,25 & 0,25 & 0,25 \\
\hline Carbonato de calcio & 1,10 & 0,95 & 0,95 & 0,95 & 0,95 & 0,95 & 1,15 & 0,95 \\
\hline Cloruro de sodio & 0,20 & 0,20 & 0,20 & 0,20 & 0,20 & 0,20 & 0,20 & 0,20 \\
\hline Ácido propiónico & 0,05 & 0,05 & 0,05 & 0,05 & 0,05 & 0,05 & 0,05 & 0,05 \\
\hline Antioxidante & 0,05 & 0,05 & 0,05 & 0,05 & 0,05 & 0,05 & 0,05 & 0,05 \\
\hline Lisina & 0,18 & 0,45 & 0,28 & 0,45 & 0.25 & 0,45 & 0,18 & 0,45 \\
\hline Metionina & 0,11 & 0,06 & 0,10 & 0,10 & 0,15 & 0.10 & 0,15 & 0,10 \\
\hline Formol & 0.05 & 0,05 & 0,05 & 0,05 & 0,05 & 0,05 & 0,05 & 0,05 \\
\hline Indicadores & \multicolumn{8}{|c|}{ Composición química de las dietas } \\
\hline Materia seca $(\%)$ & 87 & 86 & 86 & 85 & 86 & 85 & 86 & 85 \\
\hline Proteína cruda (g) & 201,30 & 183,60 & 201,30 & 184,70 & 202,40 & 184,50 & 203,60 & 184,30 \\
\hline EM (MJ/kg) & 12,55 & 13,05 & 12,55 & 12,97 & 12,51 & 12,97 & 12,59 & 12,97 \\
\hline Fibra cruda (\%) & 4,02 & 3,39 & 4,02 & 3,93 & 4,16 & 3,98 & 4,12 & 4,03 \\
\hline Calcio (\%) & 1 & 0,95 & 1 & 0,99 & 1 & 0,99 & 1,02 & 0,99 \\
\hline Fósforo (\%) & 0,49 & 0,47 & 0,40 & 0,48 & 0,49 & 0,47 & 0,42 & 0,48 \\
\hline
\end{tabular}

I, fase inicial (0-4 semanas); II, fase de crecimiento-ceba (5-8 semanas).

${ }^{1}$ Pre-mezcla de vitaminas y minerales; donde $1 \mathrm{~kg}$ de alimento contiene las vitaminas siguientes: A (10000 UI); D3 (2000 UI); E (10 mg), $\mathrm{K}_{3}(2 \mathrm{mg}) ; \mathrm{B}_{1}(1 \mathrm{mg}) ; \mathrm{B}_{2}(5 \mathrm{mg}) ; \mathrm{B}_{6}(2 \mathrm{mg}) ; \mathrm{B}_{12}(15 \mathrm{mg}) ; \mathrm{B}_{3}(125 \mathrm{mg}) ; \mathrm{B}_{5}(10 \mathrm{mg}) ; \mathrm{B}_{9}(0,25 \mathrm{mg})$ y Biotina $(0,02 \mathrm{mg})$ y micro-elementos minerales: Selenio (0,10 mg); Hierro (40 mg); Cobre (12 mg); Zinc (120 mg), Mg (100 mg); Yodo (2,50 mg) y Cobalto (0,75 mg). 
diferencias en la conversión de alimento, en ninguna de las dos fases, en los grupos (tabla II).

\section{DISCUSIÓN}

El mayor consumo total y en la fase de crecimientoceba se obtuvo en la dieta con semillas tostadas de $C$. cajan a $120^{\circ} \mathrm{C}$ por 12 min (tabla II). Aunque, no se determinaron los FAN, Belmar y Nava (2015) plantearon que los alcaloides, lectinas vegetales y glucosinolatos, reducen la palatabilidad y el consumo voluntario de los animales monogástricos, entre ellos las aves. Es importante destacar que los FAN termolábiles (inhibidores de proteasas y las fitohemaglutinas) de las semillas, se desnaturalizan con el tostado. Existen contradicciones en cuanto al tiempo y temperatura de procesamiento de las semillas de leguminosas, para eliminar los FAN, sin afectar la calidad del alimento. Quicazán y Caicedo (2012) determinaron que es posible degradar el inhibidor de tripsina en semillas de soya remojadas y escaldadas, para la elaboración de bebidas, con tratamiento térmico, a $80^{\circ} \mathrm{C}$. Mientras, Ramos et al. (2004) determinaron que a $100{ }^{\circ} \mathrm{C}$ se destruyeron los FAN, lo que no sucedió a $80^{\circ} \mathrm{C}$, pero se redujo el valor biológico de la proteína. Hirigoyen et al (2011) determinaron que la cocción de soya en agua, durante 15 minutos de hervor, es suficiente para la inactivación de los factores antinutricionales. De Luna (2006) expuso que se necesita un tiempo óptimo de temperatura, humedad y duración del tratamiento a las semillas, donde el tos- tado, la elevación de temperatura a más de $100^{\circ} \mathrm{C}$, por medio de vapor, remuevan los inhibidores de tripsina y desnaturalicen las proteínas.

El tratamiento térmico excesivo daña mayor cantidad de proteínas, disminuye el contenido de aminoácidos disponibles, reduce la digestibilidad de la proteína y afecta la calidad de la dieta (Ramos et al., 2004 y Elizalde et al., 2009), como pudo suceder con la aplicación de calor en seco, por el mayor tostado en los tratamientos a $130^{\circ} \mathrm{C}$ por $15 \mathrm{~min}$ y $140^{\circ} \mathrm{C}$ por $18 \mathrm{~min}$. Además, resulta importante destacar que $C$. cajan posee bajos niveles de FAN, según Rivero y Romero (2014), quienes detectaron sólo 27,55 unidades de tripsina inhibida, donde la cocción eliminó el 87\% de anti-tripsina. Los resultados del consumo por animal fueron similares a los de Polyana et al. (2014) que obtuvieron los más altos valores durante toda la crianza, en pollos de engorde con dietas que contenían, entre $5-10 \%$ de inclusión, de $2626,08-2640,84 \mathrm{~g}$ y la mayor conversión $(2,43)$ con el $5 \%$, hasta los 56 días de edad. Los resultados también coincidieron con los de Zambrano y Zambrano (2014) quienes recomendaron utilizar el 5\% de inclusión de C. cajan, para obtener los mejores resultados productivos. El peso vivo final y la ganancia de peso vivo más altas se obtuvieron con el 6\% de inclusión de harina de semillas tostadas de C. cajan a $120^{\circ} \mathrm{C}$ por 12 min (tabla 2). Esto se pudo deber, a la mayor disponibilidad de nutrientes, por el menor tiempo y temperatura de tostado.

\begin{tabular}{|c|c|c|c|c|c|c|}
\hline Indicadores & Control & $120^{\circ} \mathrm{C}+12 \mathrm{~min}$ & $130^{\circ} \mathrm{C}+15 \mathrm{~min}$ & $140^{\circ} \mathrm{C}+18 \mathrm{~min}$ & $\mathrm{EE}( \pm)$ & Significación $(p)$ \\
\hline \multicolumn{7}{|c|}{ Consumo de alimento } \\
\hline I & 1981,14 & 2029,14 & 2006 & 1948,86 & 28,59 & 0,4220 \\
\hline II & $5114,30^{b}$ & $5715,70^{a}$ & 5370 ab & $5174,30^{b}$ & 75,90 & 0,0200 \\
\hline Consumo total & $7095,40^{\mathrm{b}}$ & $7744,90^{\text {a }}$ & 7376 ab & 7123,10 ab & 104,54 & 0,0321 \\
\hline \multicolumn{7}{|c|}{ Comportamiento productivo } \\
\hline Peso final, $\mathrm{g}$ & $3105^{b}$ & $3566,43^{a}$ & $3085^{b}$ & $3175^{b}$ & 45,57 & 0,0004 \\
\hline \multicolumn{7}{|c|}{ Ganancia promedio diaria de peso vivo, $\mathrm{g}$} \\
\hline I & $45,79^{b}$ & $50,94^{a}$ & $46,05^{\mathrm{b}}$ & $45,03^{b}$ & 0,60 & 0,0001 \\
\hline II & $63,32^{b}$ & $74,64^{\mathrm{a}}$ & $62,35^{b}$ & $66,58^{\text {ab }}$ & 0,69 & 0,0084 \\
\hline En la crianza & $54,55^{b}$ & $62,79^{a}$ & $54,20^{\mathrm{b}}$ & $55,80^{\mathrm{b}}$ & 0,63 & 0,0004 \\
\hline \multicolumn{7}{|c|}{ Conversión de alimento } \\
\hline I & 1,44 & 1,33 & 1,44 & 1,44 & 0,01 & 0,0602 \\
\hline II & 3,09 & 2,83 & 4,10 & 2,84 & 0,02 & 0,0730 \\
\hline Total (56 días) & 2,32 & 2,20 & 2,43 & 2,28 & 0,01 & 0,0656 \\
\hline \multicolumn{7}{|c|}{ Eficiencia en el uso de la EM del aporte (EM del aporte/GMD (MJ/g)) } \\
\hline 1 & 0,27 & 0,25 & 0,27 & 0,28 & - & - \\
\hline II & 0,21 & 0,17 & 0,21 & 0,19 & - & - \\
\hline \multicolumn{7}{|c|}{ Eficiencia en el uso de la PC del aporte (PC del aporte/GMD (g/g)) } \\
\hline I & 4,40 & 3,95 & 4,40 & 4,52 & - & - \\
\hline II & 2,90 & 2,47 & 2,96 & 2,77 & - & - \\
\hline $\begin{array}{l}a, b y c \text { Letras igua } \\
\text { l, fase inicial }(0\end{array}$ & $\begin{array}{l}\text { n los súper-ín } \\
\text { manas); II, fas }\end{array}$ & $\begin{array}{l}\text { S no difieren signifi } \\
\text { crecimiento-ceba }\end{array}$ & $\begin{array}{l}\text { tivamente, para } \\
\text {-8 semanas). }\end{array}$ & & & \\
\hline
\end{tabular}


Mukhtar et al. (2013) obtuvieron menor peso vivo final por pollo $(2129,38 \mathrm{~g})$ y ganancia de peso vivo acumulada por animal $(1967,50 \mathrm{~g})$, con $20 \%$ de inclusión de harina de C. cajan. Al Hafiz et al. (2013) utilizaron la decorticación del grano, para su incorporación en la dieta de pollos de engorde. Ellos obtuvieron menores valores en el comportamiento productivo de los animales y la mayor ganancia de peso en la crianza la lograron con $12 \%$ de inclusión de la harina de C. cajan (2085 g). El comportamiento productivo también fue superior al de Chambilla (2012), con 2536,90 g de ganancia acumulada en la etapa final, donde se utilizó $15 \%$ de harina de C. cajan. Las principales diferencias en los resultados se pudieron deber a la forma de utilización del grano y su tratamiento, para reducir los FAN y mejorar el uso de los nutrientes. La conversión (tabla II) fue similar a la que obtuvo Labrador y Andara (2012), de 1,31 , en cuatro semanas de crianza, con el uso de entre 20-30\% de harina de C. cajan, lo que indicó una favorable eficiencia en el uso de los nutrientes. Trompiz et al. (2011) obtuvieron favorables parámetros productivos con el uso de $20 \%$ de harina de semillas de C. cajan. La conversión para 5\% de inclusión, fue de 1,54.

Los buenos resultados productivos se debieron a que todas las dietas cubrieron los requerimientos nutricionales. Con la mayor eficiencia en el uso de la energía y la proteína, para la ganancia de peso que se obtuvo con las semillas tostadas a $120^{\circ} \mathrm{C}$ por $12 \mathrm{~min}$, se confirmó que fue posible potenciar el valor nutritivo de la harina con ese tratamiento térmico. Amaefule et al. (2011) demostraron que en dietas con C. cajan para pollos de ceba donde se logró suplementar con lisina y metionina, como sucedió en este experimento, permiten obtener buenos resultados productivos y una inclusión de la harina, hasta del 40\%. Akande et al. (2010) demostraron que el rostizado de las semillas de C. cajan no produjo una variación importante en los nutrientes, en particular la proteína cruda, pero si incrementó la presencia de los aminoácidos arginina, ácido aspártico, treonina, serina, ácido glutámico, glicina, alanina, leucina y tirosina. Esto justifica la importancia de realizar un tostado correcto, para incrementar la disponibilidad de aminoácidos, en función de mejorar el comportamiento productivo de los pollos de engorde.

Se demostró que es posible mejorar el comportamiento productivo de pollos de ceba, en inicio y crecimiento-ceba, con la sustitución del $6 \%$ de la soya y el maíz de la ración por harina de semillas tostadas de $C$. cajan a $120^{\circ} \mathrm{C}$ por $12 \mathrm{~min}$.

\section{BIBLIOGRAFÍAS}

Akande, K.E.; Abubakar, M.M.; Adegbola, T.A.; Bogoro, S.E. and Doma, U.D. 2010. Chemical evaluation of the nutritive quality of pigeon pea (Cajanus cajan (L.) Millsp.). Int J Poult Sci, 9: 63-65.

Al Hafiz, A.H.; Elshiek, O.Y. and Gibril, S. 2013. Effect of feeding graded levels of decorticated pigeon pea (Cajanus Cajan) seeds on broiler chicks performance. J Appl Ind Sci, 1: 7-10.

Amaefule, K.U.; Ukpanah, U.A. and lbok, A.E. 2011. Performance of starter broilers fed raw pigeon pea [Cajanus cajan (L.) Millsp.] seed meal diets supplemented with lysine and or methionine. Int J Poult Sci, 10: 205-211.
AOAC. 2012. Official Methods of Analysis (19th) Ass Off Anal Chem. Arligton. VA. Washington. D.C. http://www.worldcat.org/title/official-methods-of-analysis-of-aoac-international/oclc/855542981? referer=di\&ht=edition (29/05/2015).

ASERCA. 2015. Maíz y soya en Chicago (CBOT). Reporte de precios. Agencia de Servicios a la Comercialización y Desarrollo de Mercados Agropecuarios. Secretaría de Agricultura, Ganadería, Desarrollo Rural, Pescay Alimentación (SAGARPA), México, disponible en: http://www. infoaserca. gob.mx (02/05/2015).

Barboza, Y. 2012. Diseño de alimentos potencialmente funcionales sobre la base de productos tradicionales. Tesis de Doctor en Ciencias en Biociencias y Ciencias Agroalimentarias. Universidad de Córdoba. España. 161 pp. http://helvia.uco.es/xmlui/bitstream/handle/10396/8240/2012000000643.pdf? sequence=1 (03/12/2015).

Belmar, C.R. y Nava, R.M. 2015. Factores antinutricionales en la alimentación de animales monogástricos. $15 \mathrm{pp}$. http://www.ucv.ve/ fileadmin/user_upload/facultad_agronomia/Producion_Animal/ Alimentacion_Animal/Metabolitos_secundarios.pdf (31/05/2015).

Bracho, E.H.; Rivero, N. y Romero, G. 2014. Inhibición de antitripsina en leguminosas: caraota (Phaseolus Vulgaris L.), quinconcho (Cajanus cajan L.), tapirama (Phaseolus lunatus L.) y frijol bayo (Vigna Unguiculata L.). Rev Digiciencia UDEFA, 3: 21-29.

Chambilla, C.E. 2012. Efecto de tres niveles de harina de semilla de gandul (Cajanus cajan L. Millsp), en el crecimiento de pollos parrilleros de la línea Ross 308 en el cantón Santa Fe de la provincia Caranavi. Tesis de Ingeniero Agrónomo. Universidad Mayor de San Andrés. La Paz. Bolivia, 107 pp. http://www.sidalc.net/cgi-bin/wxis. exe/?lsisScript=cidab.xis\&method=post\&formato $=2$ \&cantidad $=1$ \&ex presion $=m f n=019328(30 / 10 / 2015)$.

De Luna, J.A. 2006. Valor nutritivo de la proteína de soya. Rev Inv Cienc. Univ Aut Aguascalientes. Sept-Dic: 29-34.

Elizalde, De D.A.; Portilla, Y.P. y Chaparro D.C.C. 2009. Factores antinutricionales en semillas. Fac Cien Agrop, 7: 45-54.

Herrera, G.S.M.; Savón, L.; Lon-Wo, E.; Gutiérrez, O. y Herrera, M. 2014. Inclusión de harina de hojas de Morus alba: su efecto en la retención aparente de nutrientes, comportamiento productivo y calidad de la canal de pollos cuello desnudo. Rev Cubana Cienc Agríc, 48: 259-264.

Herrera, G.S.M. 2014. Caracterización y manejo de un sistema de alimentación alternativo en pastoreo, para pollos cuello desnudo heterocigotos. Tesis de Doctor en Ciencias Veterinarias. Instituto de Ciencia Animal. Universidad Agraria de La Habana. Cuba. pp. 165.

Hirigoyen, C.; Bratschi, S.; Furtado, G.; Arias, A.; González, R. y Bauza, R. 2011 . Caracterización química del grano de soja sometido a diferentes tratamientos de desactivación. Efecto del cocimiento en agua. Resúmenes del simposio. UDELAR. Uruguay. pp. 205.

Labrador, J. y Andara, J. 2012. Incorporación del quinchoncho (Cajanus cajan) en raciones alimenticias para pollos de engorde. Rev Cientif FCV-LUZ, 1: 27-28.

León, R.; Angulo, I. y Jaramillo, M. 2011 . Caracterización química y valor nutricional de granos de leguminosas tropicales para la alimentación de aves. Rev Zoot Trop, 11: 151-170.

Mukhtar, A.; Mukhtar, A.E. and Amal, O. 2013. Response of broiler chicks fed on diets containing graded levels of pigeon pea (Cajanus cajan) seeds supplemented with essential caraway oil. J Curr Res Sci, 1: 109-112.

Mula, M.G and Saxena, K.B. 2010. Liffing the level of awareness on Pigeon pea - A global perspective. International Crops Research Institute for the Semi-Arid Tropics, Patancheru, India. pp. 540. http:// oar.icrisat.org/193/ (7/11/2015).

Navarro, C.L; Restrepo, D.M. y Pérez, M.J. 2014. El guandul (Cajanus cajan) una alternativa en la industria de los alimentos. Rev Biotec Sect Agrop, 12: 197-206. 
Polyana, A.D.; Maciel, M.P.; Rocha, L.F.B.; Albuquerque, L.C.; Martins, L.F.S.; Batista, D.S. e Santana, V.H. de M. 2014. Feijão guandu cru na alimentação de frangos caipiras criados em sistema semi-intensivo. Rev Pesq Agropec Bras, 49: 737-744.

Quicazán, M. y Caicedo, L. 2012. Inactivación del inhibidor de tripsina, durante el tratamiento térmico de bebidas de soya. Rev Vitae, 19: 337-339.

Ramos, N.; Lúquez, J. y Eyherabide, G. 2004. Calidad de la harina de soja sometida a distintos tratamientos térmicos para inactivar los factores antinutricionales. Unidad Integrada Balcarce. Argentina. Tecnología de procesos: 681-683. http://www.acsoja.org.ar/images/ $\mathrm{cms} /$ contenidos/615_b.pdf (26/12/2015).

Rufini, M. 2014. Eficiência de estirpes de Bradyrhizobium spp. em simbiose com guandu cvs Fava-Larga E Anão em condições diversas. Tese de Doutor em Ciência do Solo. Universidade Federal de Lavras. Brasil.79p. http://repositorio.ufla.br/handle/1/1721 (10/12/2015).
Sourokou, S.S. 2014. Performances zootechnico-economiques des poulets de chair (COBB500) nourris aux rations a base de la farine des graines de la variete verte de bissap (Hibiscus sabdariffa, Linn.) au Sénégal. These Docteur en Medecine Veterinaire. Universite Cheickh Anta Diop de Dakar. Sénégal. 140 pp. http://www.beep.ird.fr/collect/eismv/ index/assoc/TD14-1.dir/TD14-1.pdf (22/12/2015)

Trómpiz, J.; Rincón, H.; Fernández, N.; González, G.; Higuera, A. y Colmenares, C. 2011. Parámetros productivos en pollos de engorde alimentados con grano de quinchoncho durante fase de crecimiento. Rev Fac Agr (LUZ), 28: 565-575.

Zambrano, F.R.F. y Zambrano, M.J.R. 2014. Inclusión de harina de frejol de palo (Cajanus cajan - L, Millsp) en el alimento de pollos de engorde y su efecto en parámetros productivos. Tesis de Médico Veterinario. Escuela Superior Politécnica Agropecuaria de Manabí. 76 pp. 